\title{
Comparative kinetics of cesium from whole-lake, limnocorrals, and laboratory-scale experiments
}

\author{
T.G. Hinton, J.E. Pinder ${ }^{1}$, F.W. Whicker ${ }^{1}$, L. Marsh, J. Joyner, D. Coughlin, \\ Y. Yi and J. Gariboldi
}

Savannah River Ecology Laboratory, University of Georgia, Aik En, SC 29801, U.S.A.

${ }^{1}$ Radiological Health Sciences, Colorado State University, Fort Collins, CO 80523, U.S.A.

\begin{abstract}
Understanding the processes governing a contaminant's transport is difficult in uncontrolled field experiments; however, controlled laboratory experiments are often contrived and may quickly loose realism. Knowing how well small-scaled, laboratory experiments mimic those from full-scale field manipulations is of fundamental importance. We compared the loss of cesium from a lake's water column at four different scales: 1) an 11 ha lake; 2) five, 1.2-m diameter limnocorrals; 3) four, 30-cm diameter limnocorrals; and 4) four, 30-cm diameter laboratory mesocolumns. Limnocorrals were installed in 1 to $2 \mathrm{~m}$ of water and at two different areas within an 11 ha lake. All limnocorrals were sealed to the lake sediments, allowed free exchange with the air, but isolated a column of the lake's water. Laboratory mesocolumns were plastic cylinders, closed on one end, and had $15 \mathrm{~cm}$ of sediments placed in them, topped with $90 \mathrm{~cm}$ of lake water. The monomictic lake had a mean and maximum depth of 1.6 and 4 $\mathrm{m}$, respectively. Stable Cs was added to the epilimnion of the whole lake, and to the limnocorrals and mesocolumns. The loss rate of cesium from the water column of each system was compared over time. The data are useful for determining how well small-scaled experiments simulate water/sediment exchange processes occurring in wholelakes.
\end{abstract}

\section{INTRODUCTION}

Experiments to address a contaminant problem can be designed at numerous scales, ranging from laboratory test tubes to manipulations of entire ecosystems. Understanding the processes governing a contaminant's environmental transport is often difficult in uncontrolled field experiments; yet, smallscaled, controlled laboratory experiments are often contrived and may quickly loose realism. Knowing how well the data generated from smaller-scaled experiments mimic those from full-scale field manipulations is fundamental to science. A disadvantage of single, large-scale experiments (for example, Cs retention in a lake) is the lack of replication. Depending on the question asked, some statisticians would argue that a whole-lake experiment has a sample size of one. Smaller-scaled limnocorrals and mesocosoms placed within the lake or laboratory avoid this problem in that they can be replicated and the variation among them characterized. The use of replicated, smaller-scaled experimental units could greatly facilitate the rigorous testing of hypotheses that are difficult to address in unreplicated designs conducted in whole-lakes. Replication also allows the use of more powerful statistical tests such as analysis of variance. But how well do the smaller-scaled experimental units mimic whole-lake processes?

We conducted an experiment to compare the loss of stable cesium from a lake's water column following an acute spike, at four different scales: 1) whole-lake; 2) five, 1.2-m diameter limnocorrals; 3) four, 30-cm diameter limnocorrals; and 4) four, 30-cm diameter laboratory mesocolumns. The data are useful for determining how well small-scaled experiments simulate water/sediment exchange processes occurring in whole-lakes.

\section{METHODS}

\subsection{Whole Lake}

Pond 4 is an 11-ha impoundment with mean and maximum depths of 1.6 and $4 \mathrm{~m}$, respectively. Oxygen and conductivity profiles indicate that periods of stratification into epilimnetic and hypolimnetic layers occurs during the summer months. Pond 4 is located in the lower coastal plain of South Carolina, USA. 
Sediments are predominantly of a sandy texture. Textural analyses of 16 randomly collected sediment samples indicated mean $( \pm S D)$ percent sand, silt, and clay of $52 \pm 7,21 \pm 5$ and $22 \pm 10$, respectively.

\subsection{Stable ${ }^{133}$ Cs Addition}

A total of $6029 \mathrm{~g}$ of $\mathrm{CsCl}$ ( $99 \%$ purity) was added to the $1.8 \times 10^{5} \mathrm{~m}^{3}$ of water in Pond 4 over a 6-hour period on August 1, 1999. The stable $\mathrm{CsCl}$ solution was added as 691 separate injections, each of $43 \mathrm{ml}$ volume, and at a water depth of $1 \mathrm{~m}$. The injection points were spaced at the intersections of a $10 \mathrm{~m} \times 10$ $\mathrm{m}$ grid across the open water sections of the pond. Navigation to injection points was performed using a real-time, differentially corrected Global Positioning System with a nominal accuracy of $1 \mathrm{~m}$. The CsCl solution was pumped from a boat into the backwash of an outboard motor.

The limnocorrals and laboratory mesocolumns received stable Cs additions after the whole-lake was spiked.

\subsection{Limnocorrals}

Limnocorrals are flexible, plastic cylinders deployed within a lake that isolate a column of water and the associated sediments. The column of water can freely interact with the atmosphere or underlying sediments, yet the column of water remains isolated from the rest of the lake. Limnocorrals were made of clear, $0.045-\mathrm{cm}$ thick, PVC plastic, with a grommetted floating collar and bottom flaps that allowed a SCUBA diver to anchor and seal the column to the lake sediments. Five limnocorrals of 1.2-m diameter and four smaller ones of $30-\mathrm{cm}$ diameter were placed in the lake at a water depth of about $130 \mathrm{~cm}$, prior to the addition of stable Cs to the whole-lake.

\subsection{Laboratory MesoColumns}

Laboratory mesocolumns are rigid, plastic cylinders, $30-\mathrm{cm}$ in diameter, and closed on one end. Fifteen $\mathrm{cm}$ of sediments were placed in the bottom and topped with $90 \mathrm{~cm}$ of lake water. Lake water was collected for this part of the experiment prior to the addition of stable Cs. The cylinders were placed outside a building, against a west-facing wall and under the protection of the roof's overhang. Stable Cs was added to each mesocolumn after the whole-lake experiment was initiated.

\subsection{Water Sampling}

Water samples were taken routinely from the lake, limnocorrals and laboratory mesocolumns using $60 \mathrm{ml}$ syringes inserted directly into the water. After obtaining the sample, a $0.45 \mu \mathrm{m}$ filter was screwed to the end of the syringe and the water was filtered into a $20 \mathrm{ml}$ vial to which $0.2 \mathrm{ml}$ of ultrapure nitric acid was added. Samples were then assayed for stable ${ }^{133} \mathrm{Cs}$ by ICP-MS. Blanks and spiked standards were assayed routinely as part of a quality control procedure.

\subsection{Statistical Analyses}

All statistical analyses were conducted on the ln-transformed data of ${ }^{133} \mathrm{Cs}$ concentrations in water. Initial graphs of ${ }^{133} \mathrm{Cs}$ loss over time indicated that a two- to three-component exponential model fit the data. Cs concentrations, however, were unstable and varied considerably during the first five days of the experiment, particularly in the whole-lake. We thus truncated the initial data (pre day 5) and compared loss rates among the different experimental units from day 5 to 136; the laboratory mesocolumns, however, were only sampled out to day 53. After day 136, Cs concentrations in the water of the large limnocorrals actually increased, due to disturbance by birds. Double-crested cormorants (large birds with wind spans of $130 \mathrm{~cm}$ ) routinely perched on the floatation collars of the large limnocorrals. Their feces increased the $\mathrm{NH}_{4}$ concentration in the water of the large limnocorrals from 0.3 to over $5 \mathrm{ppm}$. $\mathrm{NH}_{4}$ concentrations in the whole-lake water never exceeded $0.6 \mathrm{ppm}$. The increased $\mathrm{NH}_{4}$ concentrations 
likely altered Cs dynamics in the large limnocorrals, and therefore, data collected after day 136 were discarded.

The interaction term within an analysis of covariance (ANCOVA) was used to determine if the slopes were similar among the replicates within an experimental unit (large limnocorrals, small limnocorrals, laboratory mesocolumns). An outlier was identified in both the large limnocorrals and the small limnocorrals $(p<0.001)$. In both cases, removing the outlier resulted in the remaining replicates having similar slopes $(p>0.05)$.

ANCOVA was also used to test if the mean retention curves differed across treatments (i.e. experiment units). If the interaction term of the ANCOVA was significant then individual contrasts, using Bonferroni's test on the log-transformed data, as well as non-parametric statistics (Mann-Whitney tests), were used to determine which experimental unit differed from that of another.

The In-transformed data for each replicated treatment and whole lake were also linearly regressed against time. The slopes of the regressions were used as estimates of the exponential loss rates

\section{RESULTS and DISCUSSION}

\subsection{Variation among replicates}

Variation among replicate laboratory mesocolumns and the two sizes of limnocorrals are shown in Figures 1, 2 and 3. Variation among replicates was least for the laboratory mesocolumns (Fig. 1), probably because their location along the shaded side of a building provided the most protection against mixing events caused by environmental fluctuations of wind, daily temperature extremes, and rainfall.

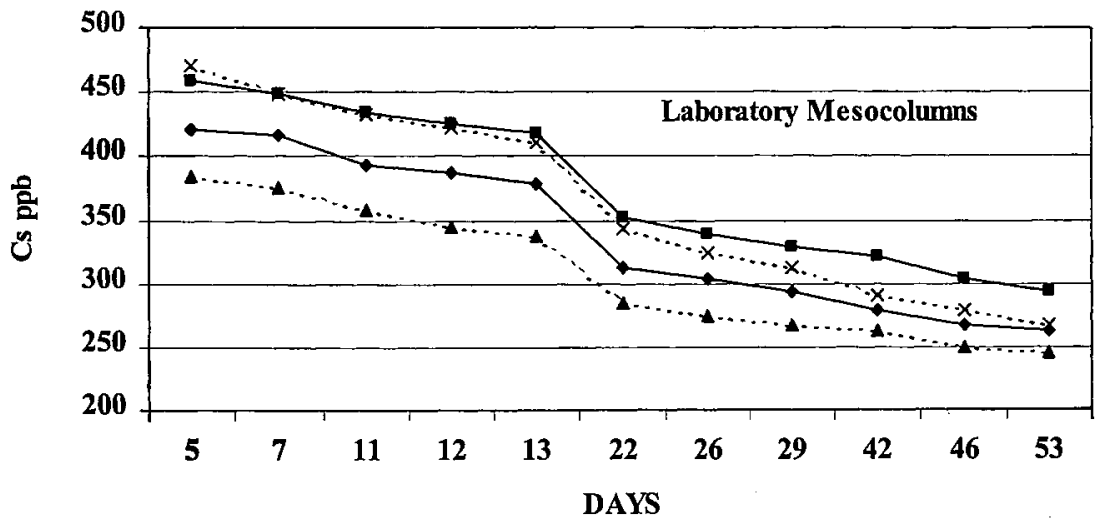

Figure 1. Variation in ${ }^{133} \mathrm{Cs}$ concentrations (ppb) over time within the water of four laboratory mesocolumns $(30 \mathrm{~cm}$ diameter). Analysis of covariance indicated similar slopes among all four replicates $(p=0.29)$.

Variation is often reduced in controlled laboratory conditions, so it is not too surprising that our most controlled experimental unit had the least variation among replicates and over time. In contrast, the whole-lake was subjected to the greatest environmental fluctuations, predominantly from wind-driven mixing events. These fluctuations caused the whole-lake to have greater daily variations in $\mathrm{Cs}$ concentrations (Fig. 4). Such wind-driven mixing events were buffered within the lake-deployed limnocorrals, which showed a moderate amount of variation from one day to the next (Fig. 4), because of the shielding from wave and wind action provided by their floatation collars.

Outliers were identified within both the large and small limnocorrals (Fig. 2 \& 3). The rapid decline of ${ }^{133} \mathrm{Cs}$ concentrations in the small limnocorral outlier, followed by Cs concentrations that were similar to those in the whole-lake, suggest that the sediment seal broke and that the water column within the limnocorral was no longer isolated. Analysis of covariance confirmed that its slope was significantly 
different $(p<0.05)$ from the remaining three replicates (Fig. 2). ANCOVA also revealed an aberrant replicate among the large limnocorrals, and its slope was significantly different from the other replicates $(\mathrm{p}<0.05)$.

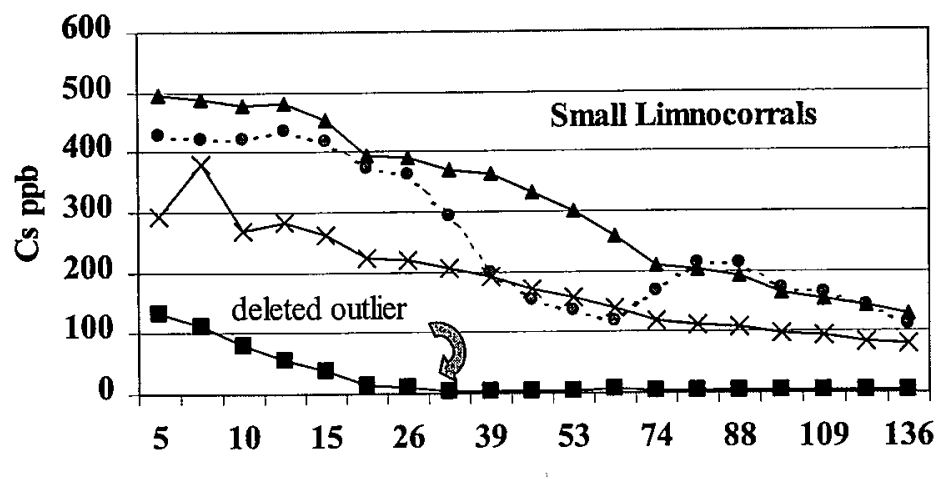

DAYS

Figure 2. Variation in ${ }^{133} \mathrm{Cs}$ concentrations (ppb) over time within the water of four small ( $30 \mathrm{~cm}$ diameter), lake-deployed, limnocorrals. The sediment seal apparently broke on one limnocorral and the data were discarded. Analysis of covariance confirmed that its slope was significantly different $(p<0.05)$ from the remaining three replicates.

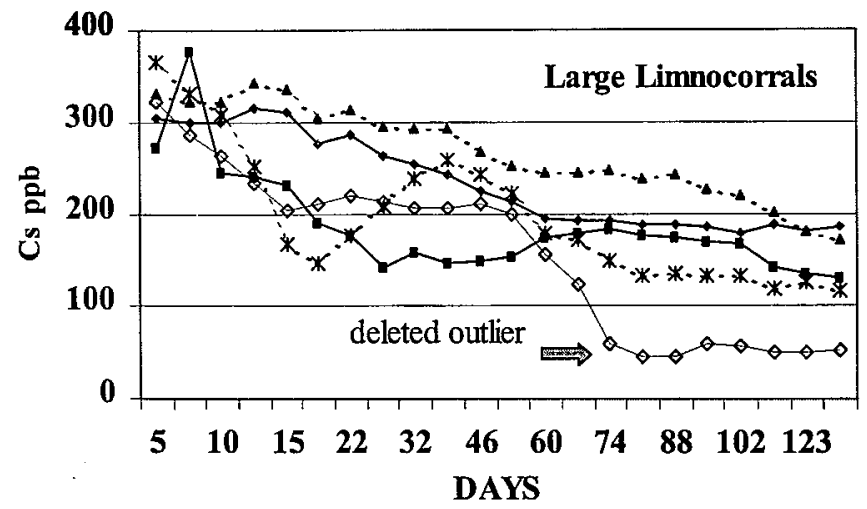

Figure 3. Variation in ${ }^{133} \mathrm{Cs}$ concentrations $(\mathrm{ppb})$ over time within the water of five large (1.2 m diameter) limnocorrals. Analysis of covariance revealed similar slopes among four of the five replicates at $p=0.09$. The fifth replicate was considered an outlier, its slope was significantly different from the others $(p<0.05)$, and was deleted.

Besides the outliers that were deleted, there was one large limnocorral and one small limnocorral that showed unusual declines in concentrations followed by unusual increases. The overall trends for these limnocorrals did not differ from those that showed no unusual changes and thus their data were included in the calculation of overall mean elimination rates. However, their aberrant data, along with the data from the limnocorrals statistically identified as outliers, suggest that temporary problems and/or permanent failures occurred in some limnocorrals. The unusual declines and increases indicate that the limnocorrals were temporarily in contact with whole-lake waters, probably due to an opening at the sediment surface caused by changes in pond levels. Once this temporary opening was resealed, equilibrium between $\mathrm{Cs}$ concentrations in the water within the limnocorral and on exchangeable sediment sites was reestablished. These temporary and permanent failures indicate the need for several replicates in limnocorral studies. 


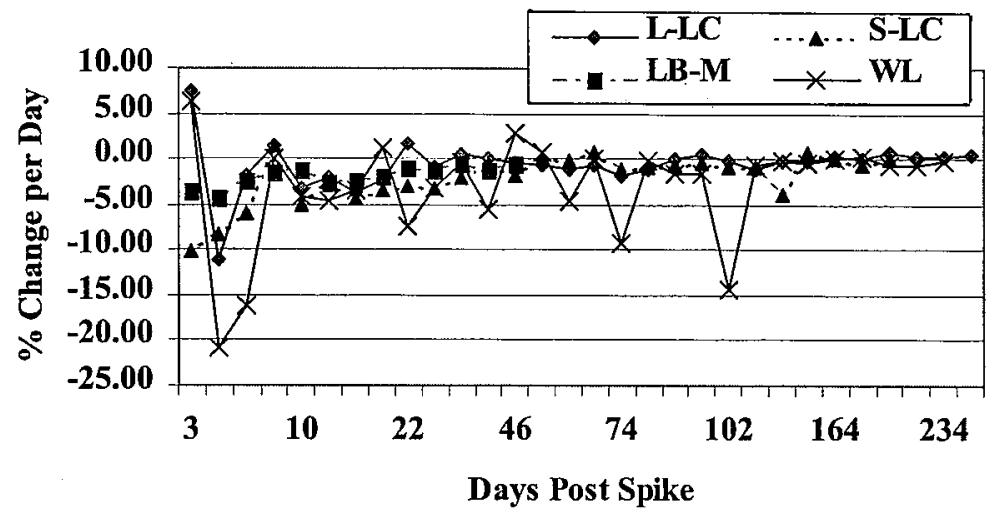

Figure 4. Percent change in ${ }^{133} \mathrm{Cs}$ concentrations per day compared among the large limnocorrals (L-LC), small limnocorrals (S-LC), laboratory mesocolumns (LB-M) and whole-lake (WL).

\subsection{Comparison of ${ }^{133} \mathrm{Cs}$ retention among experimental units}

Mean retention curves for the two different sized limnocorrals and the laboratory mesocolumns are compared to ${ }^{133} \mathrm{Cs}$ loss from the whole-lake in Figure 5. The slopes of these curves are estimates of the rate at which Cs was removed from the water column of the various experimental units. Statistical analyses indicate that ${ }^{133} \mathrm{Cs}$ retention in the two $30-\mathrm{cm}$ sized experimental units (i.e. small limnocorrals and laboratory mesocolumns) was the same as that of the whole-lake ( $p>0.2)$. Loss of ${ }^{133} \mathrm{Cs}$ from the large limnocorrals $(1.2 \mathrm{~m}$ diameter) was significantly less than all others $(\mathrm{p}<0.01)$. Table 1 converts the loss rates to half-times for each of the experimental units, and presents their retention equations and associated $\mathrm{r}^{2}$ values from the linear regressions.

The loss rates observed in the whole lake were not due to export of $\mathrm{Cs}$ in surface water outflows. There were no significant losses of surface waters from the pond during the course of the study.

The patterns of losses do not reflect simple differences in water volume to sediment area ratios among the experimental units. The volume to sediment area ratio for the large limnocorrals (1.3) was the same as the small limnocorrals, and intermediate between those for the mesocolumns $(0.9)$ and the whole lake (1.6; calculated as $1.8 \times 10^{5} \mathrm{~m}^{3} / 11 \mathrm{ha}$ ). Nor does there appear to be a relationship between Cs loss rates and surface areas of the various experimental units (whole lake sediment surface $\sim 110,000 \mathrm{~m}^{2}$; surface area of 1-ended cylinder for large limnocorral, $6.0 \mathrm{~m}^{2}$; small limnocorral, $1.3 \mathrm{~m}^{2}$; and laboratory mesocolumns, $0.9 \mathrm{~m}^{2}$ ).

TABLE 1. Linear regression results of Cs retention are shown for the large limnocorrals, small limnocorrals, laboratory mesocolumns, and whole-lake. Retention equations, $R^{2}$ values, and $95 \%$ confidence intervals about the mean $C s$ half-time are presented.

\begin{tabular}{|c|c|c|c|c|c|}
\hline Treatment & Retention Equation & $\mathbf{R}^{2}$ & $\begin{array}{c}\text { Mean } \mathbf{T}_{\mathbf{1 / 2}} \\
\text { (d) }\end{array}$ & $\begin{array}{c}\text { Lower 95\% C.I. } \\
\text { (d) }\end{array}$ & $\begin{array}{c}\text { Upper 95\% C.I. } \\
\text { (d) }\end{array}$ \\
\hline Large- LC & $\ln \mathrm{Y}=\ln (5.665)-0.0053 \mathrm{x}$ & 0.90 & 131 & 113 & 158 \\
\hline Small- LC & $\ln \mathrm{Y}=\ln (6.015)-0.0105 \mathrm{x}$ & 0.95 & 66 & 59 & 75 \\
\hline Lab-MC & $\ln \mathrm{Y}=\ln (6.086)-0.0105 \mathrm{x}$ & 0.92 & 66 & 54 & 84 \\
\hline Whole-lake & $\ln \mathrm{Y}=\ln (2.407)-0.0124 \mathrm{x}$ & 0.85 & 56 & 46 & 71 \\
\hline
\end{tabular}

Periodic measurements of $\mathrm{NH}_{4}$ in the large limnocorrals and whole-lake revealed steadily increasing $\mathrm{NH}_{4}$ levels in the large limnocorrals due to defecation by birds (see section 2.5). Ammonia readily displaces Cs from sediments. Therefore, the longer retention time that we observed in the large 
limnocorrals may be due to the re-release of sediment bound Cs rather than a slower rate of Cs loss from the water column.

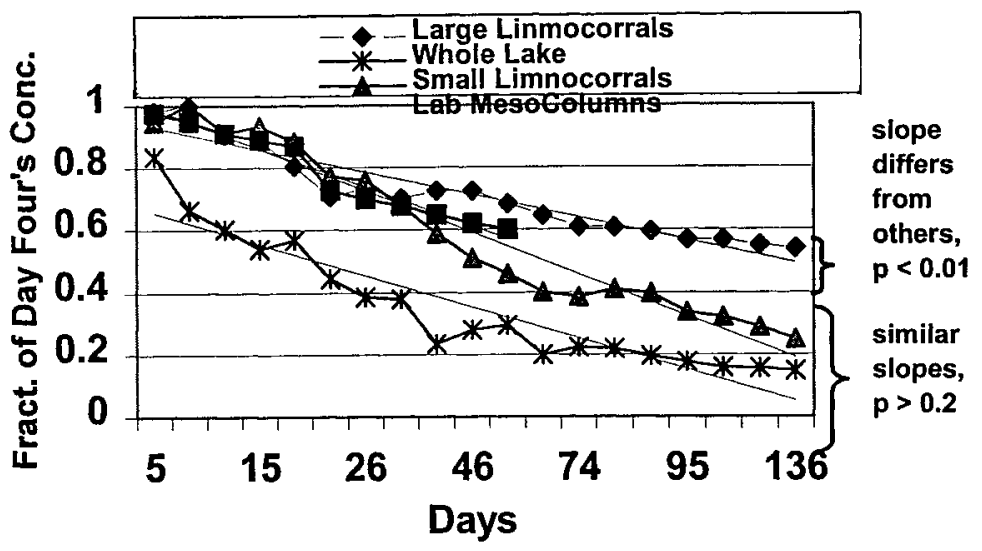

Figure 5. Mean ${ }^{133} \mathrm{Cs}$ retention in the water of large limnocorrals $(n=4)$, small limnocorrals $(n=3)$ and laboratory mesocolumns $(n=4)$ compared to that of the whole-lake $(n=1)$. Data have been normalized by presenting Cs concentrations in the water as a fraction of the concentration in each unit on day 4 of the experiment. Linear trend lines for each curve are also depicted.

\section{CONCLUSIONS}

Our expectations were not confirmed by the results of this experiment. We anticipated that the lakedeployed limnocorrals would more closely mimic the loss rate exhibited in the whole-lake, and that the larger the limnocorral the closer the data would be to that of the whole-lake. The data, however, suggested that retention within the smaller sized experimental units best mimicked the whole-lake, and that there was no difference between the laboratory mesocolumns and the field-deployed small limnocorral. Cs loss from the large units was significantly slower than all the other systems studied, perhaps due to increased $\mathrm{NH}_{4}$ concentrations brought on by fecal material from roosting birds. Overall, the smaller-scaled experimental units mimicked Cs retention in the whole-lake surprisingly well; although, it is possible that the similarity was serendipitous fortuity (i.e. random luck). It is interesting that both the laboratory mesocolumns and the small limnocorrals were of the same $30-\mathrm{cm}$ diameter. It is possible that Cs retention in these small units is driven by adsorption on to surface walls, and that their surface areas happened to promote a loss rate similar to that of the whole-lake.

Although the data are not convincing enough for us to recommend limnocorrals as a panacea to radioecological investigations in lakes, the data do indicate a real potential for their use under cautious and closely monitored conditions. Their real potential is in research where treatments or manipulations are applied to test specific hypotheses, and a replicated experimental design permits the use of powerful statistical methods such as ANOVA.

\section{Aknowledgement}

The authors gratefully acknowledge the assistance of Matt Johannsen, Jim Novak, and Brian Jackson. 